\title{
Positron Implantation Profile in Kapton
}

\author{
J. DRYZEK ${ }^{a, b, *}$ AND E. DRYZEK ${ }^{a}$ \\ ${ }^{a}$ Institute of Nuclear Physics, Radzikowskiego 152, 31-342 Kraków, Poland \\ ${ }^{b}$ Institute of Physics, University of Zielona Góra \\ Prof. Szafrana 4a, 65-516 Zielona Góra, Poland
}

\begin{abstract}
The discussion presented in the paper focuses on processes accompanying positron implantation in condensed matter. They finally constitute the positron implantation profile which generally does not exhibit the exponential behavior as it is concluded from the Monte Carlo simulation made using the EGSnrc 4.0 code. The simulation was performed for the kapton and two commonly used positron sources ${ }^{22} \mathrm{Na}$ and ${ }^{68} \mathrm{Ge} \backslash{ }^{68} \mathrm{Ga}$. New formula for the implantation profile was proposed.
\end{abstract}

PACS numbers: 78.70.--g

\section{Introduction}

The transport of an energetic positively charged positron into matter is a complex phenomenon. Broadly speaking, the interactions of positrons entering condensed matter can be divided into three stages: implantation, thermalization, diffusion and finally annihilation with a random electron. On closer inspection, definite boundaries between them cannot be strictly determined. A backscattering process due to elastic scattering at atomic nuclei accompanies the direct injection of a positron [1]. This does not allow the positron penetrate deeply into the matter. Emission of secondary electrons from the surface also accompanies the entering process [2]. At the initial stage of implantation, the channelling of a positron along the crystalline planes is also possible [3]. At very high energies, stopping is determined by radiative processes, like bremsstrahlung, resulting from interactions with the screened Coulomb potential of atomic orbital. This is less efficient for positrons than for electrons because, being positively charged, they tend to be repelled by the ion cores. A change in the direction occurs by the multiple Coulomb scattering from nuclei, scattering from electrons, Compton scattering, coherent Rayleigh scattering. At this stage, due to the ionization and relaxation of excited atoms after vacancies are created, the emission of fluorescent photons and Auger or Coster-Kronig electrons takes place. It is reflected in the emission of characteristic X-rays suitable to the implanted medium [4]. For energy of the

*corresponding author; e-mail: Jerzy.Dryzek@ifj.edu.pl 
order of hundreds $\mathrm{keV}$, mainly ionization of the $K$ shell takes place, but at lower energies the emission of the Auger electrons and ionization of $L$ shell is observed. At lower energies, typically used in positron beam experiments (up to $50 \mathrm{keV}$ ) inelastic scattering of the incident positron from free electrons is significant. This process is described by the Bhabha cross-section. At somewhat lower energies the plasma excitation and hole creation contribute to the energy-loss process. The latter gives a very strong maximum at $50-100 \mathrm{eV}$ for the stopping power of positrons, which corresponds to a minimum in the mean-free path [5]. One should emphasize that up to this point the slowing-down process is very fast, and it takes only $1 \mathrm{ps}\left(10^{-12} \mathrm{~s}\right)$ though positron penetrates a large space in the matter. Below the energy of $0.2 \mathrm{eV}$, only elastic and inelastic scattering on acoustic phonons takes place; this process is much slower, and takes only few ps. It finishes the thermalization stage, and the positron begins random walk with thermal energy. During a relatively large time of the order of 100 to $500 \mathrm{ps}$, it inspects mainly the interstitial regions; the average distance is only of the order of micrometers. In liquids and molecular materials, like polymers, during thermalization or diffusion a positron can trap an electron, and a hydrogen-like structure, called positronium, can be formed. This changes the dynamics of slowing down process. Finally, the positron annihilates with an electron and it finishes its life. The emission of annihilation quanta, mainly two emitted in opposite directions quanta having energy of about $511 \mathrm{keV}$, allows us to localize the place where it occurred. Nevertheless, one should mention that a very low fraction of positrons, less than $1 \%$, can annihilate as well during implantation and thermalization, we call this annihilation in flight [6].

The described above processes form the implantation depth profile, which is an important phenomenon, and tool in basic research, industry and medicine. Approximately, the profile decreases exponentially as a function of depth or absorber thickness, and it is described by only one physical parameter - the linear absorption coefficient - which depends generally on the cross-sections of possible scattering processes. Recent computer simulation and experimental techniques allow us to refine the exponential decay law over the original results [7] which were established over 50 years ago. For this purpose positrons are a good choice because after implantation, thermalization and then random walk they annihilate with the emission of two energetic photons of $\approx 511 \mathrm{keV}$. This allows one to track accurately the particle. Some computer codes (e.g. GEANT 4.0 [8], FOTELP [9], EGSnrc 4.0 [10]) are available which give Monte Carlo simulations for tracking energetic positrons, electron or photons entering condensed matter. In our studies we used EGSnrc 4.0 which is a general purpose package for Monte Carlo simulations of coupled transport of electrons, positrons and photons in an arbitrary geometry for particles with energies in the range of a few $\mathrm{keV}$ to several hundreds $\mathrm{GeV}$.

\section{Results and discussion}

In our calculations, we assumed a point source of positrons located in the middle of a sample of an infinite size. Positrons are randomly emitted into the 
full solid angle with energies for $\beta^{+}$decay of ${ }^{22} \mathrm{Na}$ or ${ }^{68} \mathrm{Ge} \backslash{ }^{68} \mathrm{Ga}$ sources. In both isotopes there are two branches of $\beta^{+}$decay and they were taken into account in the simulations. The position of a positron when its energy reached a value of $1 \mathrm{keV}$ was assumed to be at the end of its life. Below this energy positron penetrates the distance of less than $1 \mu \mathrm{m}$, which is much less than its total range. To obtain each implantation profile $10^{6}$ positrons were used. In Fig. 1, the normalized profile is depicted for positrons emitted from the sources ${ }^{22} \mathrm{Na}$ and ${ }^{68} \mathrm{Ge} \backslash{ }^{68} \mathrm{Ga}$ into kapton. We took into account this material because it is willingly used in the positron annihilation technique.
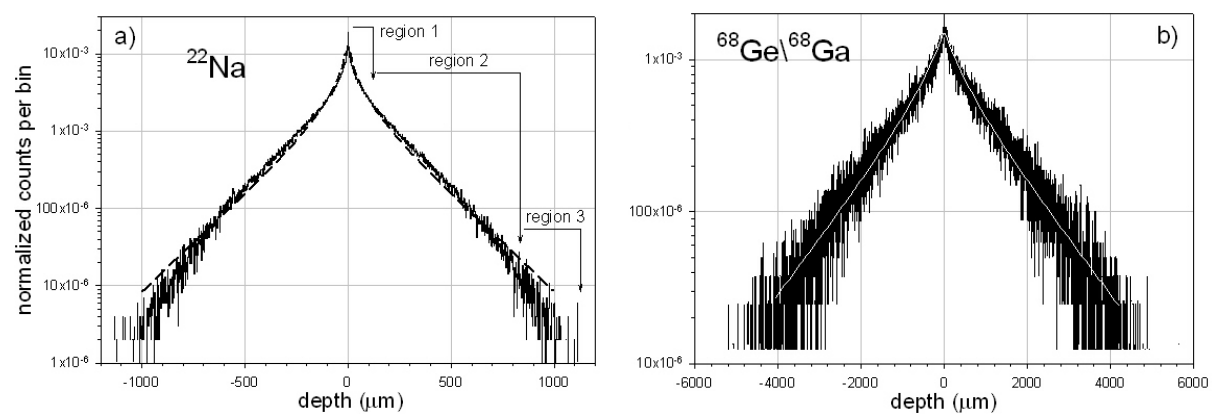

Fig. 1. The normalized implantation profile of positrons from ${ }^{22} \mathrm{Na}$ (a) and ${ }^{68} \mathrm{Ge} \backslash{ }^{68} \mathrm{Ga}$ (b) sources in kapton obtained from the Monte Carlo simulation using EGSnrs 4.0 code. The dashed line represents the results from Eq. (1). The best fit was achieved when the standard deviation of the Gaussian function in (1) was taken as quadratic function of the positron kinetic energy, see the text.

Generally, the shape of the profile is not affected by the material and in all cases, it contains the same features, see Fig. 1. Near the source, there is the fast decay region, called region 1 , where the profile falls more rapidly. In region 2 the profile is given by an exponential decay function. In region 3 the profile decreases rapidly and finally reaches the depth where no positron is able to travel. Actually, the latter is not detectable in experiments because of high background originating from other gammas and imperfect shielding of other annihilation photons that do not originate in the slit window. Nevertheless, the simulations point out the existence of the total range of the implantation profile for positrons emitted from the radioactive source. The region 1 was observed in kapton by several authors $[11,12]$.

In region 2 , by fitting the exponential decay function to the simulated profile, we were able to extract the mean penetration depth, which is the reciprocal value of the linear mass absorption coefficient. For positrons emitted from the ${ }^{22} \mathrm{Na}$ source it is equal to $147 \pm 1.2 \mu \mathrm{m}$. (We have added the uncertainty which arises from the fitting procedure.) This value corresponds well with this obtained experimentally using the depth scanning of the implantation profile (DSIP) method: 
$155 \pm 5.5 \mu \mathrm{m}$ [13]. This method allows us to scan the profile of implanted positrons into solid samples with a good accuracy [14]. The mean penetration depth for the ${ }^{68} \mathrm{Ge} \backslash{ }^{68} \mathrm{Ga}$ source extracted in the similar way from the simulated profile (Fig. 1b) is equal to $966 \pm 12 \mu \mathrm{m}$. No experimental data are available.

The value of the mean penetration depth can be helpful in determination of the total radius of the region 3 , which is approximately seven times its value. In addition, the region 1 can be characterized by the exponential behavior, on closer inspection the mean penetration depth of positrons in region 1 is about twice of that for region 2. The radius of the region 1 is about one fourth of the mean penetration depth. This allows us to characterize the implantation profile only in approximation way. To improve it we need a new formula for describing it in the whole range.

Following the suggestions of Spanel et al. [15] we argue that the shape of the implantation profile reflects not only the responsible for slowing down processes but also the shape of the energy spectrum of the implanted positrons. We have found that the implantation profile for monoenergetic positrons implanted randomly into the full solid angle in kapton exhibits almost perfect Gaussian shape. Its standard deviation or FWHM increases with increasing positron kinetic energy as a parabolic function. For small positron energies, the shape of the profile is much narrower than for higher energies. In the spectrum of the radioactive sources used, there are such low energy positrons and they contribute mainly in region 1 . The convolution of the energy spectrum of the ${ }^{22} \mathrm{Na}$ positrons with a Gaussian of standard deviation $\sigma(E)$, which depends on the positron kinetic energy, should be a good approximation for describing both the simulated and observed profiles. In our calculation we used the following relation:

$$
N(z)=\frac{N_{0}}{\sqrt{2 \pi}} \int_{0}^{E_{\max }} \frac{P(E)}{\sigma(E)} \mathrm{e}^{-z^{2} / 2 \sigma(E)^{2}} \mathrm{~d} E,
$$

where $P(E)$ represents the energy spectrum of the emitted positrons as obtained from Fermi's theory of $\beta^{+}$decay. In Fig. 1 we depict the results of the best fit of this equation to the implantation profile obtained from the Monte Carlo simulation of ${ }^{22} \mathrm{Na}$ and ${ }^{68} \mathrm{Ge} \backslash{ }^{68} \mathrm{Ga}$ positrons into kapton. The good fit for ${ }^{22} \mathrm{Na}$ was obtained when: $\sigma(E)=0.15 E+1.86 \times 10^{-3} E^{2}$, and for ${ }^{68} \mathrm{Ge} \backslash{ }^{68} \mathrm{Ga}$ $\sigma(E)=4.0 E+6.74 \times 10^{-3} E^{2}$, where $E$ is the positron kinetic energy in keV. It is apparent that while the profile in regions 1 and 2 are accurately described by this equation, the profile in region 3 is not. However, the increase in the slope in this region is noticeable. The use of Eq. (1) to describe the positron implantation profile required the assumption that the standard deviation had a quadratic dependence with respect to two parameters. These parameters were used as the free parameters in the fitting procedure, and they replaced the linear absorption coefficient, which was the single parameter used previously for describing the implantation profile. In doing this one loses the clear physical interpretation that one had using only the linear absorption coefficient. The radius of the region 3 


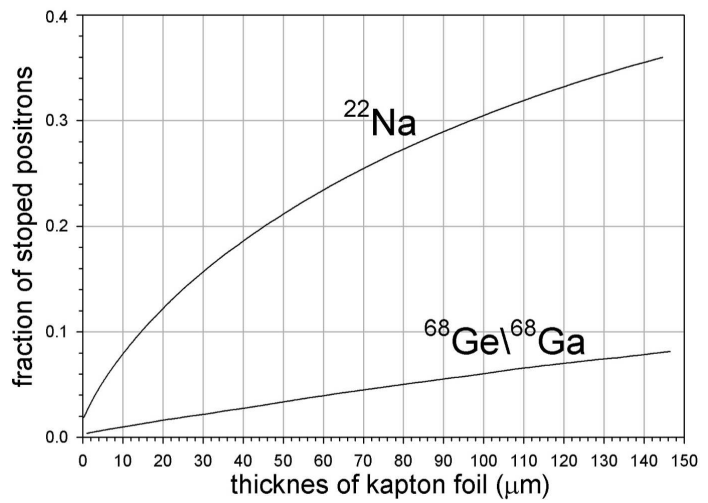

Fig. 2. The fraction of positrons stopped as a function of the thickness of the kapton foil determined from the simulated Monte Carlo profile, see Fig. 1.

(i.e. total range of the profile) and the slope in the region 1 can be expressed in a simple way via the mean penetration depth determined from region 2. For this reason, it is not necessary to reject it.

In the positron experiment the kapton foil is used as the material for the construction of the envelope which contains the source. By this reason it is interesting to know the fraction of positron stopped in the foil and do not penetrate samples. This fraction was measured by several authors [11] but now one can extract it from the simulated profiles presented in Fig. 1. In Fig. 2 we depicted it as a function of the thickness of the foil for two commonly used sources. Due to the existence of region 1 the fraction cannot be described by the simple exponential function. This corresponds with the detected experimentally profile [12]. Nevertheless, in the experiment these values are slightly larger. Due to backscattering from the sample positrons return to the kapton foil and annihilate there.

\section{Conclusions}

The computer simulations of the implantation profile for positrons emitted from the radioactive source reveal three well separated regions. Only in the regions 1 and 2 the profile can be described by the exponential decay function. In the region 3, which surrounded these regions the numbers of stopped positrons rapidly decay. For the description of the three regions the new formula (1) was proposed. The value of the mean penetration depth obtained from the region 2 corresponds well with that determined experimentally.

\section{Acknowledgments}

This research was partly supported in the frame of the program COST Action 532 by the State Committee for Scientific Research project No. 620/ E-77/SPB/COST/T-08/DWM 49/2004-2005. 


\section{References}

[1] I.K. MacKenzie, C.W. Shulte, T. Jackman, J.L. Campbell, Phys. Rev. A 7, 135 (1973).

[2] E. Jung, R. Venkataraman, S. Starnes, A.H. Weiss, Mater. Sci. Forum 255-257, 708 (1997).

[3] R. Behnish, Phys. Status Solidi 33, 375 (1969).

[4] J. Dryzek, E. Dryzek, Phys. Lett. A 320, 238 (2003).

[5] R.M. Nieminen, J. Oliva, Phys. Rev. B 22, 2226 (1980).

[6] G. Azuelos, J.E. Kitching, At. Data Nucl. Data Tables 17, 104 (1976).

[7] G.I. Gleason, I.D. Taylor, D.L. Tabern, Nucleonics 8, 12 (1951).

[8] S. Agostinelli, J. Allison, K. Amako, J. Apostolakis, H. Araujo, P. Arce, M. Asai, D. Axen, S. Banerjee, G. Barrand, F. Behner, L. Bellagamba, J. Boudreau, L. Broglia, A. Brunengo, H. Burkhardt, S. Chauvie, J. Chuma, R. Chytracek, G. Cooperman, G. Cosmo, P. Degtyarenko, A. Dell'Acqua, G. Depaola, D. Dietrich, R. Enami, A. Feliciello, C. Ferguson, H. Fesefeld, G. Folger, F. Foppiano, A. Forti, S. Garelli, S. Giani, R. Giannitrapani, D. Gibin, J.J. Gómez Cadenas, I. González, G. Gracia Abril, G. Greeniaus, W. Greiner, V. Grichine, A. Grossheim, S. Guatelli, P. Gumplinger, R. Hamatsu, K. Hashimoto, H. Hasui, A. Heikkinen, A. Howard, V. Ivanchenko, A. Johnson, F.W. Jones, J. Kallenbach, N. Kanaya, M. Kawabata, Y. Kawabata, M. Kawaguti, S. Kelner, P. Kent, A. Kimura, T. Kodama, R. Kokoulin, M. Kossov, H. Kurashige, E. Lamanna, T. Lampén, V. Lara, V. Lefebure, F. Lei, M. Liendl, W. Lockman, F. Longo, S. Magni, M. Maire, E. Medernach, K. Minamimoto, P. Mora de Freitas, Y. Morita, K. Murakami, M. Nagamatu, R. Nartallo, P. Nieminen, T. Nishimura, K. Ohtsubo, M. Okamura, S. O'Neale, Y. Oohata, K. Paech, J. Perl, A. Pfeiffer, M.G. Pia, F. Ranjard, A. Rybin, S. Sadilov, E. Di Salvo, G. Santin, T. Sasaki, N. Savvas, Y. Sawada, S. Scherer, S. Sei, V. Sirotenko, D. Smith, N. Starkov, H. Stoecker, J. Sulkimo, M. Takahata, S. Tanaka, E. Tcherniaev, E. Safai Tehrani, M. Tropeano, P. Truscott, H. Uno, L. Urban, P. Urban, M. Verderi, A. Walkden, W. Wander, H. Weber, J.P. Wellisch, T. Wenaus, D.C. Williams, D. Wright, T. Yamada, H. Yoshida, D. Zschiesche, Nucl. Instrum. Methods Phys. Res. A 506, 250 (2003); GEANT4 Web page: http://cern.ch/geant4.

[9] R.D. Ilić, J. Skvarc, A.N. Golovchenko, Radiat. Measur. 36, 83 (2003). See also R.D. Ilić, http://www.vin.bg.ac.yu/ rasa/hopa.htm, Belgrad 2003.

[10] J.A. Treurniet, D.W.O. Rogers, NRC Report PIRS-669, Oct 1999. On line: http://www.irs.inms.nrc.ca/inms/EGS_Windows/distribution.html.

[11] K. Płotowski, T.J. Panek, J. Kansy, Nuovo Cimento D 10, 933 (1988).

[12] N. Djourelov, M. Misheva, J. Phys. 8, 2081 (1996).

[13] J. Dryzek, Acta Phys. Pol. A 107, 598 (2005).

[14] J. Dryzek, Appl. Phys. A 81, 1099 (2005).

[15] L.E. Spanel, A.S. Rupaal, J.R. Patrick, Phys. Rev. B 8, 4072 (1973). 